\title{
OPTIMIZACIÓN DE LA FERTILIZACIÓN NITROGENADA PARA TRIGO DE ALTO POTENCIAL DE RENDIMIENTO EN ANDISOLES DE LA REGIÓN DE LA ARAUCANÍA, CHILE
}

\author{
Optimization of nitrogen fertilization for high-yielding potential wheat on Andisols \\ at the Araucanía Region, Chile
}

Ricardo Campillo R. ${ }^{*}$, Claudio Jobet F. ${ }^{1}$ y Pablo Undurraga D. ${ }^{2}$

\begin{abstract}
A B S T R A C T
The use of high $\mathrm{N}$ doses to enhance the expression of the yield potential in currently marketed wheat (Triticum aestivum L.) cultivars requires a careful and efficient fractionating of the total amount in order to minimize nutrient losses and improve nitrogen use efficiency (EUN). The effect of a range of annual $\mathrm{N}$ doses on the performance of high-yielding cv. Do1linco-INIA was studied in a Vilcún series soil of the Pachic Melanudans family (Andisol) in the Araucanía Region, Chile. The objective was to determine doses that optimize productivity and grain quality. Five annual $\mathrm{N}$ doses $\left(0,150,200,250\right.$ and $\left.300 \mathrm{~kg} \mathrm{ha}^{-1}\right)$ as Nitromag (CAN 27), were evaluated in a randomized complete block design with three replicates, during two successive winter wheat cropping seasons, on a soil subjected to inten-sive rotation with annuals crops. A significant effect $(\mathrm{P} \leq 0.01)$ of the $\mathrm{N}$ doses on grain production and quality was found. The optimal economic annual $\mathrm{N}$ dose (DOE) ranged between 239 and $253 \mathrm{~kg} \mathrm{ha}^{-1}$. The estimates of EUN ( $\mathrm{kg}$ of grain produced $\mathrm{kg}^{-1} \mathrm{~N}$ ) were high (38.4 and 40.3). The results confirm that high $\mathrm{N}$ requirements need to be met for the new high-yielding wheat cultivar DollincoINA, in order to achieve its full yield potential.
\end{abstract}

Key words: nitrogen level, nitrogen use efficiency, Andisols, Triticum aestivum.

\section{R E S U M E N}

\begin{abstract}
El uso de altas dosis de $\mathrm{N}$ para obtener los potenciales de rendimiento de los cultivares de trigo (Triticum aestivum L.) actualmente disponibles en el mercado, requiere un manejo cuidadoso y eficiente de la parcialización de este nutriente para minimizar sus pérdidas y mejorar la eficiencia de uso del N (EUN). En un suelo serie Vilcún de la familia de los Pachic Melanudands (Andisol) de la Región de la Araucanía, Chile, se estudió el efecto de un rango de dosis anuales crecientes de $\mathrm{N}$ sobre el cv. Dollinco-INIA, trigo alternativo de alto potencial de rendimiento, con el fin de establecer las dosis de $\mathrm{N}$ que optimizan su productividad y calidad de grano. En un diseño de bloques completos al azar con tres repeticiones se evaluaron cinco dosis anuales de $\mathrm{N}$ como Nitromag (CAN 27) (0, $150,200,250$ y $300 \mathrm{~kg} \mathrm{ha}^{-1}$ ) durante dos temporadas sucesivas, en un suelo sometido a rotaciones intensivas de cultivos anuales. Los resultados indicaron efectos significativos $(\mathrm{P} \leq 0,01)$ de la dosis de $\mathrm{N}$ sobre la productividad y calidad del grano cosechado. Las dosis óptimas económicas (DOE) anuales de N fluctuaron entre 239 y $253 \mathrm{~kg} \mathrm{ha}^{-1}$ en siembras de trigo de invierno. Las EUN fertilizante ( $\mathrm{kg}$ grano producido $\left.\mathrm{kg}^{-1} \mathrm{~N}\right)$ fueron altas $(38,4$ y 40,3$)$ y fluctuaron en rangos similares. Los resultados confirman el mayor requerimiento de $\mathrm{N}$ del cv. Dollinco-INIA para expresar su potencial de rendimiento de grano.
\end{abstract}

Palabras clave: dosis de nitrógeno, eficiencia de uso de nitrógeno, Andisoles, Triticum aestivum.

\footnotetext{
${ }^{1}$ Instituto de Investigaciones Agropecuarias, Centro Regional de Investigación Carillanca, Casilla 58-D, Temuco, Chile. E-mail: rcampill@inia.cl *Autor para correspondencia.

${ }^{2}$ Instituto de Investigaciones Agropecuarias, Centro Regional de Investigación Quilamapu, Casilla 426, Chillán, Chile. Recibido: 1 de agosto de 2006. Aceptado: 20 de diciembre de 2006.
} 


\section{INTRODUCCIÓN}

Existen variados factores que inciden en la calidad y productividad del trigo (Triticum aestivum L.) en el sur de Chile, donde el clima, la genética y el manejo del cultivo aparecen como las más relevantes. La productividad triguera nacional es la más alta del Cono Sur de América y se sustenta en el empleo de variedades de alto potencial de rendimiento, que requieren un uso intensivo de insumos como fertilizantes, enmiendas calcáreas y pesticidas (Fundación Chile, 2005).

La rentabilidad de los sistemas de producción de trigo en ambientes favorables no depende sólo de la investigación en mejoramiento genético del trigo. Las tecnologías de manejo del cultivo que incrementan la eficiencia en el uso de insumos fertilizantes son esenciales (Parodi, 2003). Los altos rendimientos son resultado de condiciones ambientales, tecnología, manejo, capital e insumos. La utilización de dosis elevadas de $\mathrm{N}$ que permitan la expresión del potencial de rendimiento de las variedades existentes en el mercado actual, requiere adicionalmente un manejo cuidadoso y eficiente de la parcialización del nutriente, con el fin de minimizar las pérdidas por lixiviación durante el desarrollo del cultivo, evitando la contaminación de napas freáticas subterráneas y su efecto nocivo sobre la salud humana y la sustentabilidad ambiental.

En comparación con otros países del Cono Sur, Chile presenta altos costos de insumos en fertilizantes y pesticidas, siendo uno de los factores de mayor incidencia en el costo total de producción (Fundación Chile, 2005). En particular, el ítem fertilizante representa un $30 \%$ de los costos directos en una situación de rendimientos promedio $\left(5 \mathrm{a} 6 \mathrm{t} \mathrm{ha}^{-1}\right)$, aumentando a un $36 \%$ en manejos de altos rendimientos (sobre $8 \mathrm{t} \mathrm{ha}^{-1}$ ). Dentro de los insumos fertilizantes utilizados en el cultivo del trigo, el fertilizante nitrogenado es normalmente el de mayor incidencia en los costos de producción (Fundación Chile, 2005).

Un mayor conocimiento acerca de los factores que determinan el uso racional del $\mathrm{N}$ permite a los productores ser más eficientes, tanto desde el punto de vista técnico como económico, en el empleo de fertilizantes a través de prácticas agronómicas y medioambientales adecuadas (Parodi, 2003). No obstante, para que los productores de trigo adopten las tecnologías que minimizan el impacto del $\mathrm{N}$ sobre el ambiente, éstas deben mantener los niveles de rendimiento y ser económicamente viables.

La eficiencia de uso del nitrógeno (EUN) fue definida por Moll et al. (1982) como el rendimiento de grano por unidad de $\mathrm{N}$ disponible ( $\mathrm{N}$ del suelo y del fertilizante). Ellos también propusieron que la EUN puede ser dividida en los componentes de eficiencia de absorción de $\mathrm{N}$ ( $\mathrm{N}$ en la planta por unidad de $\mathrm{N}$ fertilizante) y en la eficiencia de utilización de $\mathrm{N}$ (rendimiento de grano por unidad de $\mathrm{N}$ en la planta). El producto de estos dos componentes se expresa como EUN (Ortiz-Monasterio et al., 1997). La EUN en trigo se estima actualmente en 33\% (Raun y Johnson, 1999), cifra bastante menor que el 50\% que se encontraba normalmente en la literatura (Rodríguez, 1991). Varios experimentos de recuperación de ${ }^{15} \mathrm{~N}$ han informado de pérdidas del 20 a $50 \%$ del fertilizante nitrogenado en trigo, atribuidas a los efectos combinados de desnitrificación, volatilización y lixiviación (Raun y Johnson, 1999). Modificar el momento y la forma de aplicación de $\mathrm{N}$ puede inducir también un mejoramiento en la eficiencia de absorción.

En el sur de Chile hay una amplia gama de cultivares nuevos de trigo que los productores están sembrando y que requieren altas dosis anuales de $\mathrm{N}$ para expresar su productividad. Esto cobra mayor validez en la Región de La Araucanía, por la gran superficie de cultivos anuales que siembra, donde el trigo es preponderante. Durante la temporada 20042005 esta región alcanzó una superficie sembrada de 259070 ha de cultivos anuales, de las cuales el trigo alcanzó 160910 ha con un promedio de rendimiento de 4,35 $\mathrm{t} \mathrm{ha}^{-1}$ (ODEPA, 2005).

El cultivar Dollinco-INIA es un trigo de calidad panadera, semienano, de hábito de desarrollo alternativo, producido por el Programa Nacional de Trigo del Instituto de Investigaciones Agropecuarias (INIA), Centro Regional de Investigación Carillanca, Temuco. Su rendimiento promedio (cuatro temporadas) en ensayos ha fluctuado entre 7,84 y 14,46 tha $^{-1}$ en tres localidades de la zona sur (Hewstone y Jobet, 2001). Es un trigo para panificación directa, con alta cifra de sedimentación $\left(55,8 \mathrm{~cm}^{3}\right)$ y buen volumen de pan $\left(749 \mathrm{~cm}^{3}\right)$. Se recomienda para la IX y X Regiones, en siembras desde fines de abril hasta agosto (Hewstone y Jobet, 2001). En la actualidad, INIA-Carillanca está desarrollando una 
línea de investigación que busca optimizar el manejo de $\mathrm{N}$ de nuevos cultivares de trigo de alto potencial de rendimiento, como Dollinco-INIA, buscando armonizar la productividad y calidad del grano producido con el cuidado del medio ambiente.

El objetivo de este estudio fue avaluar, en un Andisol de la Región de La Araucanía sometido a una rotación intensiva de cultivos anuales, el efecto de dosis crecientes de $\mathrm{N}$ sobre el rendimiento y peso hectolitro, y establecer las dosis que optimizan la productividad y calidad de grano de un cultivar de trigo de alto potencial de rendimiento.

\section{MATERIALES Y MÉTODOS}

El estudio se realizó en el Centro Regional de Investigación (CRI) Carillanca, perteneciente al Instituto de Investigaciones Agropecuarias (INIA), comuna de Vilcún (38 $41^{\prime}$ lat. Sur, $72^{\circ} 25^{\prime}$ long. Oeste, 200 m.s.n.m.), cercana a la ciudad de Temuco, Región de La Araucanía, Chile, durante dos temporadas (2002-2003 y 2003-2004). El suelo corresponde a la serie Vilcún, miembro de la familia medial, mésica de los Pachic Melanudands (Andisol) (CIREN, 2002). Los análisis químicos de ambos sitios se realizaron en el Laboratorio de Suelos del INIA, CRI Quilamapu, Chillán. La caracteriza- ción inicial de los suelos se presenta en el Cuadro 1, de acuerdo a las metodologías establecidas en Sadzawka et al. (2006). Ambos sitios presentaron niveles de fertilidad adecuados, sin limitaciones por acidez y con un bajo nivel de $\mathrm{N}$ inicial.

El diseño utilizado fue de bloques completos al azar con tres repeticiones, en parcelas de $10 \mathrm{~m}^{2}(2 \times 5 \mathrm{~m})$. Se evaluaron cinco tratamientos consistentes en dosis anuales de N: 0, 150, 200, 250 y $300 \mathrm{~kg} \mathrm{ha}^{-1}$, utilizando el fertilizante nitrogenado comercial (FNC) Nitromag (27-0-0-5\% MgO-7\% CaO). Es un fertilizante conocido como CAN 27, es decir, nitrato de amonio cálcico con $27 \%$ de N, mezclado con cal dolomítica. Sin embargo, por tener un contenido de $\mathrm{N}$ superior a $22 \%$ está considerado como un fertilizante de reacción moderadamente ácida (Suárez, 1994).

Un mes previo a la siembra, el suelo recibió un encalado de neutralización con $750 \mathrm{~kg} \mathrm{ha}^{-1} \mathrm{de} \mathrm{CaCO}_{3}$ (cal Soprocal), para prevenir daños por los efectos acidificantes del FNC (Campillo y Sadzawka, 2006). El FNC se parcializó en cuatro oportunidades, entre paréntesis se indica el correspondiente código decimal para los estados de crecimiento de los cereales según Zadoks et al. (1974): a la siembra $\left(Z_{0}\right)$; tres macollas $\left(Z_{23}\right)$; fines de macolla $\left(Z_{30}\right)$, y dos nudos

Cuadro 1. Caracterización química del suelo $(0-20 \mathrm{~cm})$ previo al establecimiento de los experimentos. Carillanca, temporadas 2002-2003 y 2003-2004.

Table 1. Chemical characterization of soil $(0-20 \mathrm{~cm})$ before experiment establishment. Carillanca, seasons 2002-2003 and 2003-2004.

\begin{tabular}{lcc}
\hline Característica química & $\begin{array}{c}\text { Temporada } \\
\mathbf{2 0 0 2 - 2 0 0 3}\end{array}$ & $\begin{array}{c}\text { Temporada } \\
\mathbf{2 0 0 3 - 2 0 0 4}\end{array}$ \\
\hline P Olsen, mg kg-1 & 14,0 & 16,0 \\
Materia orgánica, \% & 17,0 & 16,0 \\
N inorgánico, mg kg & 15,0 & 14,0 \\
pH agua, $1: 2,5$ & 6,3 & 6,0 \\
Ca intercambiable, cmol $\mathrm{kg}^{-1}$ & 10,80 & 6,69 \\
Mg intercambiable, cmol $\mathrm{kg}^{-1}$ & 0,97 & 0,65 \\
Na intercambiable, cmol $\mathrm{kg}^{-1}$ & 0,13 & 0,19 \\
$\mathrm{~K}$ intercambiable, cmol $\mathrm{kg}^{-1}$ & 0,32 & 0,39 \\
Suma de bases intercambiables, $\mathrm{cmol}_{+} \mathrm{kg}^{-1}$ & 12,22 & 7,92 \\
Al intercambiable, cmol $\mathrm{kg}^{-1}$ & 0,02 & 0,04 \\
CICE, cmol $\mathrm{kg}^{-1}$ & 12,24 & 7,96 \\
Saturación de Al, \% & 0,2 & 0,5 \\
Zn, mg kg $^{-1}$ & 0,68 & 0,85 \\
$\mathrm{~B}, \mathrm{mg} \mathrm{kg}^{-1}$ & 0,26 & 0,62 \\
$\mathrm{~S}, \mathrm{mg} \mathrm{kg}$ & 18,28 & 15,75 \\
\hline
\end{tabular}

CICE: capacidad de intercambio catiónico efectiva. 
$\left(Z_{32}\right)$. A la siembra siempre se aplicaron $40 \mathrm{~kg} \mathrm{ha}^{-1} \mathrm{de}$ $\mathrm{N}$. El saldo de cada dosis de $\mathrm{N}$ se dividió proporcionalmente en los restantes tres estados de Zadoks. Así, por ejemplo, la dosis anual de $250 \mathrm{~kg} \mathrm{ha}^{-1}$ de $\mathrm{N}$ fue parcializada de la siguiente forma: $40 \mathrm{~kg} \mathrm{ha}^{-1}\left(\mathrm{Z}_{0}\right) ; 70$ $\mathrm{kg} \mathrm{ha}^{-1}\left(\mathrm{Z}_{23}\right) ; 70 \mathrm{~kg} \mathrm{ha}^{-1}\left(\mathrm{Z}_{30}\right)$ y $70 \mathrm{~kg} \mathrm{ha}^{-1}\left(\mathrm{Z}_{32}\right)$.

En ambas temporadas las siembras se efectuaron con una máquina sembradora de ensayos (Planet Junior, Allen Co, New Jersey, USA). Tres semanas antes de la siembra se colectaron muestras de suelo para la caracterización química del suelo. Las fechas de siembra fueron el 12 de junio de 2002 y 14 de mayo de 2003. El trigo sembrado fue el cv. Dollinco-INIA (hábito alternativo), teniendo como precultivo avena (Avena sativa L.) para grano. La dosis de semilla fue $180 \mathrm{~kg} \mathrm{ha}^{-1} \mathrm{y}$ se desinfectó con triticonazole en dosis de $200 \mathrm{~g} \mathrm{~L}^{-1}$ (Real $200 \mathrm{SC}, 300$ $\left.\mathrm{cm}^{3} 100 \mathrm{~kg}^{-1}\right)$.

Se estableció una fertilización base para el cultivo que compensara cualquier limitación nutricional que no fuera el N; en el surco de siembra se aplicó una fertilización base (por ha) de $240 \mathrm{~kg}$ de $\mathrm{P}_{2} \mathrm{O}_{5}$ (superfosfato triple), $55 \mathrm{~kg}$ de $\mathrm{K}_{2} \mathrm{O}, 55 \mathrm{~kg}$ de $\mathrm{S}$ y 45 $\mathrm{kg}$ de $\mathrm{MgO}$ (sulpomag), 2,5 $\mathrm{kg}$ de $\mathrm{B}$ (boronatrocalcita) y $2,2 \mathrm{~kg}$ de $\mathrm{Zn}$ (sulfato de zinc). Esta fertilización a la siembra se complementó con una aplicación al voleo de $50 \mathrm{~kg}$ de $\mathrm{K}_{2} \mathrm{O}$ y $18 \mathrm{~kg}$ de $\mathrm{S}$ (sulfato de potasio). Finalmente, en el estado $Z_{30}$ se adicionaron $65 \mathrm{~kg}$ de $\mathrm{K}_{2} \mathrm{O}$ (muriato de potasio).

En la primera temporada el control químico de malezas (AFIPA, 2006) se realizó con metsulfuronmetil en dosis de $600 \mathrm{~g} \mathrm{~L}^{-1}$ (Ally, $8 \mathrm{~g} \mathrm{ha}^{-1}$ ), MCPA amina en dosis de $750 \mathrm{~g} \mathrm{~L}^{-1}\left(750 \mathrm{~cm}^{3} \mathrm{ha}^{-1}\right)$ y clodinafop-propargil en dosis de $240 \mathrm{~g} \mathrm{~L}^{-1}$ (Topik 240 EC, $300 \mathrm{~cm}^{3} \mathrm{ha}^{-1}$ ). En la segunda temporada, el control de las malezas se realizó con iodosulfuronmetil-sodio en dosis de $50 \mathrm{~g} \mathrm{~kg}^{-1}$ (Hussar $20 \mathrm{WG}$, $\left.300 \mathrm{~g} \mathrm{ha}^{-1}\right)$. Durante la temporada 2002-2003 $\left(\mathrm{Z}_{30}\right)$ se utilizaron los fungicidas prochloraz + carbendazima en dosis de $300 \mathrm{~g} \mathrm{~L}^{-1}+80 \mathrm{~g} \mathrm{~L}^{-1}$ (Sportak $40 \mathrm{EC}$ $1 \mathrm{~L} \mathrm{ha}^{-1}$ ) y benomil en dosis de $500 \mathrm{~g} \mathrm{~kg}^{-1}$ (Benlate, $\left.0,5 \mathrm{~kg} \mathrm{ha}^{-1}\right)$. Esta misma aplicación se repitió en los estados $Z_{32}, Z_{39}$ y $Z_{69}$ para prevenir la presencia de enfermedades foliares. En la temporada 2003-2004 las enfermedades foliares se controlaron con los fungicidas prochloraz y benomil. En ambas temporadas se utilizó el fitorregulador cloruro de clormequat + cloruro de colina en dosis de $460 \mathrm{~g} \mathrm{~L}^{-1}+320$ $\mathrm{g} \mathrm{L}^{-1}$ (Cycocel extra, $3 \mathrm{~L} \mathrm{ha}^{-1}$.) en el estado $Z_{28}$.
Durante el período de crecimiento del cultivo se colectaron muestras de suelo para la determinación del contenido de humedad (base peso seco) a 0-20 y $20-40 \mathrm{~cm}$ de profundidad. Las cosechas de ambos experimentos se realizaron el 13 de febrero de 2003 y 6 de febrero de 2004, respectivamente. Se determinó el rendimiento de grano con $14 \%$ de humedad base peso seco (HBPS), peso hectolitro, índice de cosecha (\%), porcentaje de $\mathrm{N}$ foliar, porcentaje de proteína $(\% \mathrm{~N}$ x 5,7$)$, gluten húmedo y $\mathrm{N}$ absorbido en el grano y follaje. En esta oportunidad sólo se analizaron los resultados de rendimiento de grano y peso hectolitro de trigo. Los datos de rendimiento de grano y peso hectolitro se sometieron al análisis de varianza (ANDEVA) y las medias de tratamientos al test de Tukey $(\mathrm{P} \leq 0,05)$ en los casos en que correspondía (SAS Institute, 1990).

Para establecer las dosis óptimas físicas y económicas derivadas del experimento, se ajustó un polinomio de segundo grado a los resultados de rendimientos de grano del trigo Dollinco-INIA: $Y=a+$ $b X+c X^{2}$ donde $Y$ es el rendimiento en $\mathrm{t} \mathrm{ha}^{-1} ; X$ es la dosis de $\mathrm{kg} \mathrm{ha}^{-1}$ mientras que $a, b$ y $c$ son parámetros de la regresión (Volke, 1982; Rebolledo, 1999). De esta manera, fue posible estimar la dosis óptima física (DOF) de producción de trigo y su correspondiente rendimiento máximo. Asumiendo un costo por unidad de N (FNC) y un valor por tonelada de trigo, se calculó la relación de precios insumo/ producto y fue posible determinar la dosis óptima económica (DOE) de $\mathrm{N}$ para producción de trigo y su respectivo rendimiento óptimo económico. Se utilizó el criterio económico de capital ilimitado con la restricción de una tasa de retorno mínimo de $50 \%$ (Volke, 1982). Como la tasa de retorno es la ganancia que el productor obtiene por el dinero invertido, en los cálculos realizados una tasa del $50 \%$ significa que el productor obtiene a la cosecha un ingreso neto de $\$ 50$ por cada $\$ 100$ invertidos.

\section{RESULTADOS Y DISCUSIÓN}

La información climática de la localidad de Carillanca (pluviometría y evaporación) durante el año 2002 se presenta en la Figura 1. A partir del segundo semestre del año las precipitaciones se incrementaron notoriamente superando el promedio histórico, principalmente entre los meses de septiembre y noviembre. En general, el año 2002 mostró una precipitación abundante que se prolongó durante toda la primavera, asegurando así un 


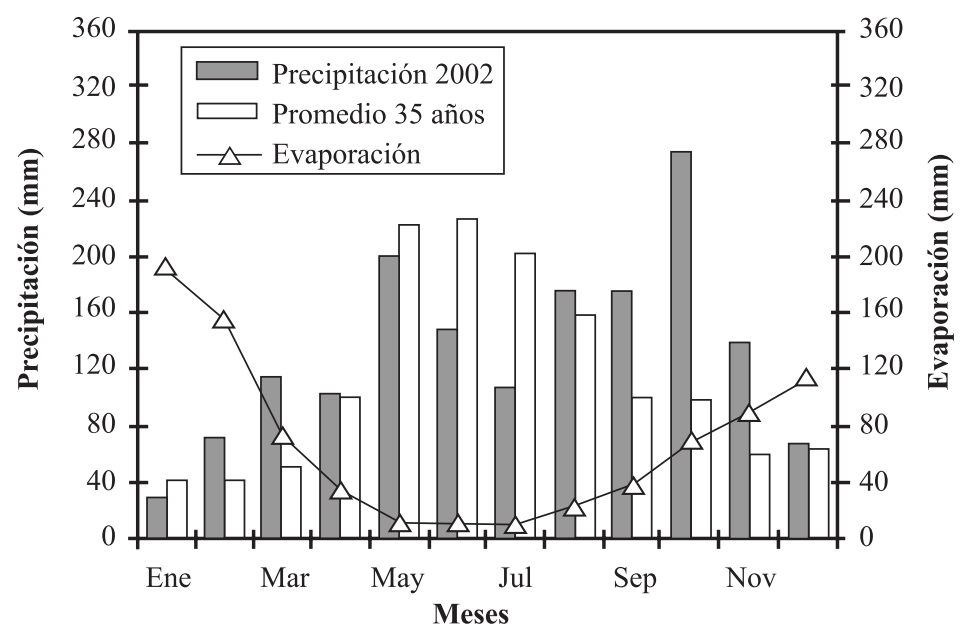

Figura 1. Antecedentes del clima en la localidad Carillanca durante el año 2002. Figure 1. Climatic information of Carillanca locality during year 2002.

buen abastecimiento hídrico para el desarrollo del cultivo de trigo.

La evolución del contenido de humedad del suelo a dos profundidades durante la temporada agrícola 2002-2003 se presenta en la Figura 3. No hubo restricciones de humedad en el suelo en ambas estratas durante todo el período de desarrollo del cultivo. En cambio, durante el año 2003, a pesar que octubre fue seco (Figura 2) se acumuló una precipi- tación anual de $1237 \mathrm{~mm}$, pero con una primavera bastante fría, y a partir de noviembre se comenzó a manifestar un déficit hídrico que afectó el suministro de agua para el desarrollo normal del cultivo de trigo. Esta situación se puede visualizar mejor con la evolución del contenido de agua almacenada en el suelo (Figura 4). Las constantes hídricas del Andisol en ambas temporadas fueron $77 \%$ para capacidad de campo (CC) y $36,5 \%$ para punto de marchitez permanente (PMP) (Figuras 3 y 4).

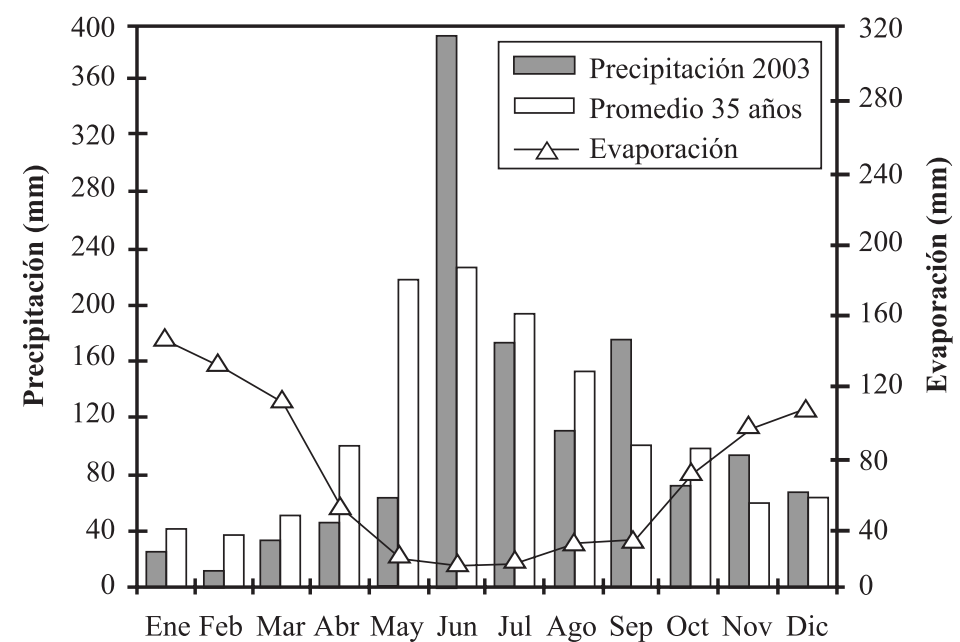

Meses

Figura 2. Antecedentes del clima en la localidad Carillanca durante el año 2003.

Figure 2. Climatic information of Carillanca locality during year 2003. 
A partir de noviembre de 2003 la humedad del suelo disminuyó fuertemente en ambas estratas, observándose en diciembre un severo déficit de humedad principalmente en el estrato superficial $(0-20 \mathrm{~cm})$, mientras que durante enero y febrero de 2004 el suelo ya se encontraba en PMP (Figura 4). La disponibilidad de agua es fundamental para asegurar un buen llenado del grano de trigo. Sin duda que este desbalance hídrico afectó en alguna medida la plena expresión del potencial de rendimiento del cv. Dollinco-INIA. Rouanet et al. (2001) encontraron que la disminución de la humedad aprovechable del suelo desde octubre en adelante habría afectado negativamente los procesos de translocación de fotosintatos y $\mathrm{N}$ al grano de trigo cv. DalcahueINIA, sembrado en la comuna de Nueva Imperial, Región de la Araucanía.

La dosis de $\mathrm{N}$ afectó significativamente $(\mathrm{P}<0,05)$ el rendimiento de grano del cv. Dollinco-INIA sembrado en ambas temporadas, presentando un coeficiente de variación bastante bajo (3,1 a 4,0\%). Los rendimientos de grano en ambas temporadas (Cuadros 2 y 3 ) aumentaron en función de la dosis de $\mathrm{N}$ y fluctuaron entre 3,05 (Testigo $\sin \mathrm{N}$ ) y $10,63 \mathrm{t} \mathrm{ha}^{-1}$ $\left(300 \mathrm{~kg} \mathrm{ha}^{-1} \mathrm{~N}\right)$. En ambos casos, las producciones más eficientes estuvieron ligadas a las aplicaciones anuales más altas de $\mathrm{N}$.

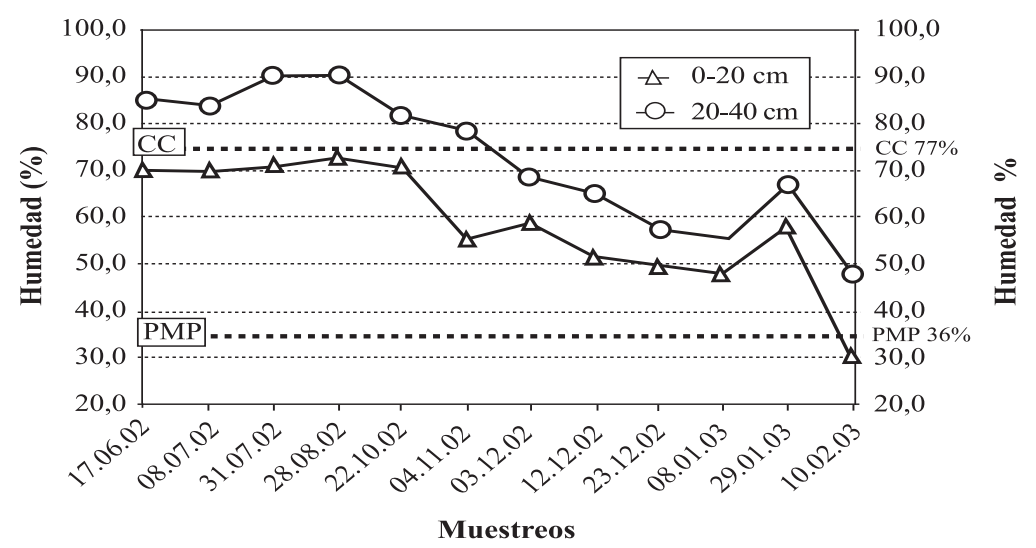

Figura 3. Evolución del contenido de humedad (\%) del suelo con trigo. Carillanca, 2002-2003. Figure 3. Evolution of moisture content (\%) of soil with wheat. Carillanca, 2002-2003.

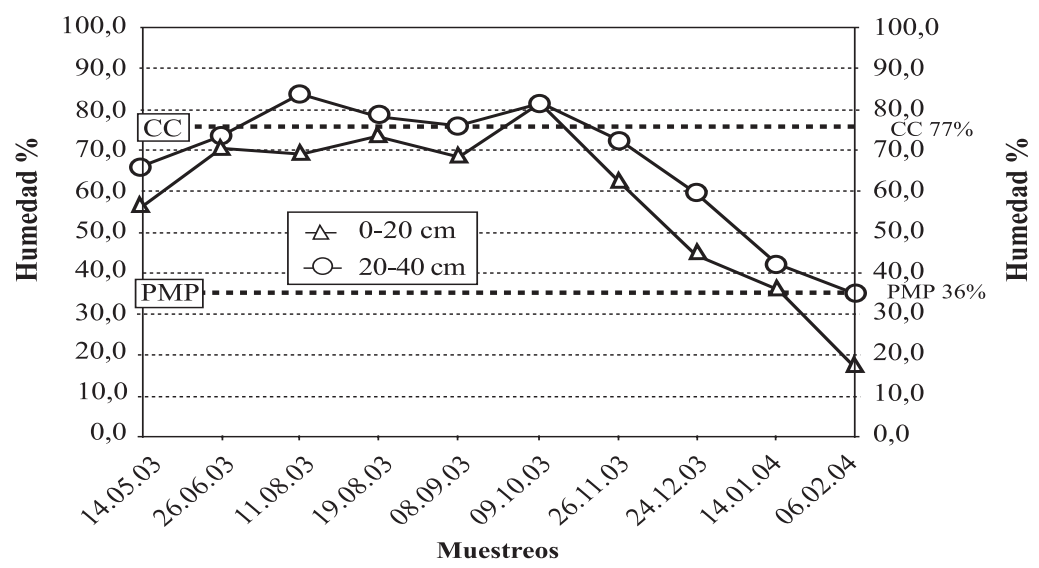

Figura 4. Evolución del contenido de humedad (\%) del suelo con trigo. Carillanca, 2003-2004. Figure 4. Evolution of moisture content (\%) of soil with wheat. Carillanca, 2003-2004. 
Las mayores producciones de trigo se registraron durante la primera temporada, superando en casi 1 $\mathrm{t}$ ha ${ }^{-1}$ a las producciones de la segunda temporada. Es probable que la primavera del año 2003 con temperaturas notoriamente más bajas que la temporada anterior, haya disminuido la capacidad de mineralización de $\mathrm{N}$ del suelo, lo cual unido al déficit hídrico que ocurrió a partir de noviembre de 2003 (Figura 2), afectó negativamente la producción de grano de trigo.

La eficiencia de uso de N (EUN), definida como la producción de grano por unidad de $\mathrm{N}$ suministrado (aportado por el suelo y el fertilizante) (Moll et al., 1982; Ortiz-Monasterio et al., 1997) disminuyó en ambas temporadas (Cuadros 2 y 3), en función del incremento de la dosis anual aplicada.

Las EUN calculadas para el cv. Dollinco-INIA fueron altas y relativamente estables en ambas temporadas, fluctuando entre 57,3 y 34,8 (2002-2003) y 52,7 y 31,3 (2003-2004). La dosis estudiada de 250 $\mathrm{kg} \mathrm{ha}^{-1}$ de $\mathrm{N}$ fue de alta eficiencia productiva $\mathrm{y}$ económica, puesto que estuvo asociada a las dosis óptimas económicas estimadas a partir del modelo cuadrático (Cuadros 2 y 3 ).

Cuadro 2. Efecto de la dosis de $\mathrm{N}$ sobre el rendimiento de grano y eficiencia de uso de $\mathrm{N}$ (EUN) de trigo cv. Dollinco-INIA sembrado en invierno. Carillanca, 2002-2003.

Table 2. Effect of $\mathbf{N}$ level on grain yield and nitrogen use efficiency (EUN) of wheat cv. Dollinco-INIA sowed at winter. Carillanca, 2002-2003.

\begin{tabular}{|c|c|c|c|}
\hline $\begin{array}{l}\mathrm{N} \text { anual } \\
\mathrm{kg} \mathrm{ha}^{-1}\end{array}$ & $\underset{\text { t ha }^{-1}}{\text { Rendimiento grano }}$ & $\begin{array}{c}\text { Ajuste cuadrático } \\
\text { t ha }^{-1}\end{array}$ & $\begin{array}{c}\text { EUN } \\
\text { kg grano } \text { kg }^{-1} \mathrm{Ns}\end{array}$ \\
\hline 0 & $3,48 \mathrm{~d}$ & 3,52 & \\
\hline 150 & $8,74 \mathrm{c}$ & 8,59 & 57,3 \\
\hline 200 & $9,63 \mathrm{bc}$ & 9,56 & 47,8 \\
\hline 250 & $9,79 \mathrm{ab}$ & 10,18 & 40,7 \\
\hline 300 & $10,63 \mathrm{a}$ & 10,43 & 34,8 \\
\hline $\mathrm{CV}$ & 4,0 & & \\
\hline Prueba de F & $* *$ & & \\
\hline
\end{tabular}

Medias con letras diferentes indican diferencias significativas según Test de Tukey $(\mathrm{P}<0,05)$.

Ns: N suministrado por el suelo y el fertilizante.

$\mathrm{CV}$ : coeficiente de variación; ** $(\mathrm{P}<0,01)$.

Cuadro 3. Efecto de la dosis de $\mathrm{N}$ sobre el rendimiento de grano y eficiencia de uso de $\mathrm{N}$ (EUN) de trigo cv. Dollinco-INIA sembrado en invierno. Carillanca, 2003-2004.

Table 3. Effect of $N$ level on grain yield and nitrogen use efficiency (EUN) of wheat cv. Dollinco-INIA sowed at winter. Carillanca, 2003-2004.

\begin{tabular}{|c|c|c|c|}
\hline $\begin{array}{c}\mathrm{N} \text { anual } \\
\mathrm{kg} \mathrm{ha}^{-1}\end{array}$ & $\begin{array}{c}\text { Rendimiento grano } \\
\mathbf{t ~ h a}^{-1}\end{array}$ & $\begin{array}{c}\text { Ajuste cuadrático } \\
\mathbf{t ~ h a}^{-1}\end{array}$ & $\begin{array}{c}\text { EUN } \\
\text { kg grano kg-1 Ns }\end{array}$ \\
\hline 0 & $3,05 \mathrm{~d}$ & 3,09 & \\
\hline 150 & $8,15 \mathrm{c}$ & 7,90 & 52,7 \\
\hline 200 & $8,63 \mathrm{bc}$ & 8,77 & 43,9 \\
\hline 250 & $9,04 \mathrm{ab}$ & 9,26 & 37,0 \\
\hline 300 & $9,54 \mathrm{a}$ & 9,39 & 31,3 \\
\hline $\mathrm{CV}$ & 3,1 & & \\
\hline Prueba de F & $* *$ & & \\
\hline
\end{tabular}

Medias con letras diferentes indican diferencias significativas según el Test de Tukey $(\mathrm{P}<0,05)$.

Ns: N suministrado por el suelo y el fertilizante; $C V$ : coeficiente de variación; ** $(\mathrm{P}<0,01)$. 
Las EUN calculadas para el cv. Dollinco-INIA en este trabajo, están por sobre los valores normalmente señalados en otras investigaciones. Díaz et al. (2002) encontraron en Andisoles de la precordillera de la Región del Bío-Bío, que la EUN fue menor a $6 \mathrm{~kg}_{\text {grano }} \mathrm{kg}^{-1} \mathrm{~N}$ aplicado con dosis de $240 \mathrm{~kg} \mathrm{ha}^{-1} \mathrm{de}$ $\mathrm{N}$ anual en siembras invernales de trigo. Por su parte, Ortiz-Monasterio et al. (1997), comparando la EUN de 10 cultivares de trigo producidos por CIMMYT (Centro Internacional de Mejoramiento del Maíz y el Trigo) en Sonora, México, encontraron que las EUN promedio fueron de $35 \mathrm{~kg}$ grano $\mathrm{kg}^{-1} \mathrm{~N}$ con dosis de $150 \mathrm{~kg} \mathrm{ha}^{-1}$ de $\mathrm{N}$ y de $18 \mathrm{~kg}$ grano $\mathrm{kg}^{-1} \mathrm{~N}$ con $300 \mathrm{~kg} \mathrm{ha}^{-1}$ de $\mathrm{N}$.

Se ha establecido que la aplicación de $\mathrm{N}$ fertilizante puede incrementar tanto el rendimiento como el contenido de proteína del grano (Fowler et al., 1990). La eficiencia de utilización de $\mathrm{N}$ es mayor cuando la respuesta en rendimiento al $\mathrm{N}$ es alta. Por ello, esta eficiencia generalmente es alta con dosis bajas de $\mathrm{N}$ y disminuye según se incrementa la dosis de $\mathrm{N}$ aplicada (Campbell et al., 1977; Clarke et al., 1990).

Para estimar las dosis de $\mathrm{N}$ óptimas, tanto físicas como económicas, derivadas del experimento en ambas temporadas, a los resultados de rendimientos de grano del cv. Dollinco-INIA se le ajustó un polinomio de segundo grado: $Y=a+b X+c X^{2}$, donde $Y$ es el rendimiento en $\mathrm{t} \mathrm{ha}^{-1} ; X$ es la dosis anual de $\mathrm{N}$ en $\mathrm{kg} \mathrm{ha}^{-1}$, mientras que $a, b$ y $c$ son parámetros de la regresión. De esta manera, fue posible estimar la dosis óptima física (DOF) de producción de trigo y su correspondiente rendimiento máximo (Figuras 5 y 6 ).

Asumiendo un costo por unidad de N (FNC) y un valor por tonelada de trigo, se calculó la relación de precios insumo/producto y fue posible determinar la dosis óptima económica (DOE) de N para producción de trigo y su respectivo rendimiento óptimo económico. Se utilizó el criterio económico de capital ilimitado con la restricción de una tasa de retorno mínimo de un 50\% (Volke, 1982; Rebolledo, 1999).

Es importante recordar que la urea aplicada en los Andisoles se hidroliza enzimáticamente y con gran rapidez. Posteriormente, su comportamiento en el suelo es similar a cualquier otra forma de $\mathrm{N}$ amoniacal (Campillo y Rodríguez, 1984), razón por la cual, $\mathrm{y}$ en el corto plazo, tanto la urea como el FNC experimentan transformaciones similares en el proceso de nitrificación. Sólo con fines de comparación de costos de fuentes nitrogenadas presentes en el mercado nacional, también se calcularon los valores relativos a la urea (Cuadro 4), puesto que es el fertilizante que normalmente presenta la unidad de $\mathrm{N}$ más económica del mercado.

En el caso del FNC, durante la primera temporada (2002-2003) la DOF alcanzó $311 \mathrm{~kg} \mathrm{ha}^{-1}$, con una DOE de $253 \mathrm{~kg} \mathrm{ha}^{-1}$ y un rendimiento óptimo de $10,21 \mathrm{t} \mathrm{ha}^{-1}$. Sin embargo, como en la segunda temporada los rendimientos alcanzados por el trigo

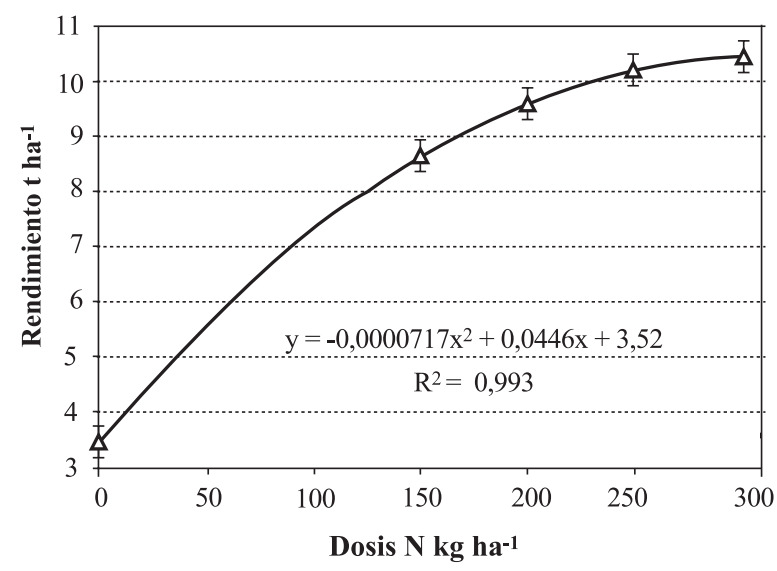

Figura 5. Incremento del rendimiento de trigo según dosis anual de N. Carillanca, 2002-2003.

Figure 5. Increase of wheat yield according annual N level. Carillanca, 2002-2003.

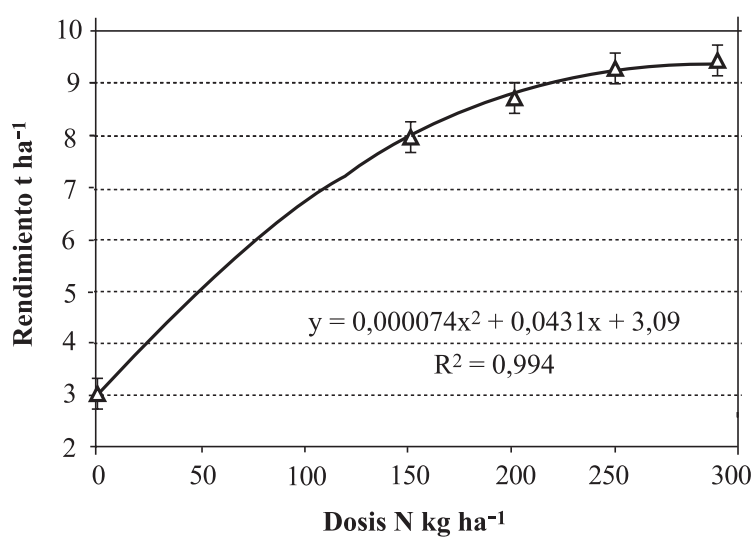

Figura 6. Incremento del rendimiento de trigo según dosis anual de N. Carillanca, 2003-2004.

Figure 6. Increase of wheat yield according annual $\mathbf{N}$ level. Carillanca, 2003-2004. 
Dollinco-INIA fueron menores, tanto las DOF (292 $\left.\mathrm{kg} \mathrm{ha}^{-1}\right)$, DOE $\left(239 \mathrm{~kg} \mathrm{ha}^{-1}\right)$ y el rendimiento óptimo calculados $\left(9,18 \mathrm{t} \mathrm{ha}^{-1}\right)$ también presentaron una disminución en sus valores. En cuanto a la comparación de estos fertilizantes nitrogenados (Cuadro 4), dado que el costo por unidad de N como urea es el más económico del mercado, es posible aplicar al cultivo DOE de N más altas (274 y $255 \mathrm{~kg} \mathrm{ha}^{-1}$ ) que con $\mathrm{FNC}$, alcanzando también rendimientos óptimos levemente mayores $\left(10,35\right.$ y 9,29 $\left.\mathrm{t} \mathrm{ha}^{-1}\right)$.

De acuerdo al análisis de suelo realizado a la cosecha del cereal (Cuadro 5), no hubo cambios significativos en los parámetros de acidez del suelo ( $\mathrm{pH}$ en agua, Ca y Al de intercambio, y saturación de Al) atribuibles a las dosis anuales de $\mathrm{N}$ aplicadas como FNC, por el efecto corrector del encalado de neutralización que se aplicó en la presiembra del cereal. Es importante recordar que cuando se aplican ferti- lizantes de reacción acidificante en suelos que inicialmente no presentan limitaciones por acidificación, siempre debe considerarse un encalado de neutralización. Esta práctica posibilita el uso de fertilizantes de reacción ácida o moderadamente ácida como el FNC, neutralizándolos con aplicaciones moderadas de carbonato de calcio (de presiembra) para prevenir eventuales daños al desarrollo y producción del cultivo.

Respecto al peso del hectolitro de grano de trigo (Cuadro 6), en la primera temporada los valores mayores fueron iguales y estuvieron asociados a las dosis de 250 y $300 \mathrm{~kg} \mathrm{ha}^{-1}$ de $\mathrm{N}$ anual, sin presentar diferencias $(\mathrm{P}<0,05)$ entre ellas. Durante la segunda temporada, cuando las producciones de grano fueron inferiores, las diferencias de esta variable de respuesta fueron menores y sólo se diferenciaron las dosis más extremas. En ambas temporadas los

Cuadro 4. Optimización de las dosis de $\mathrm{N}$ en trigo cv. Dollinco-INIA con dos fuentes nitrogenadas.

Table 4. Optimization of $\mathrm{N}$ levels on wheat cv. Dollinco-INIA with two nitrogen sources.

\begin{tabular}{|c|c|c|c|c|c|c|}
\hline $\begin{array}{c}\text { Fuente de } \\
\text { N }\end{array}$ & $\begin{array}{c}\text { Unidad de } \mathbf{N} \\
\$\end{array}$ & $\begin{array}{c}\text { Precio trigo } \\
\$ \mathrm{t}^{-1}\end{array}$ & $\begin{array}{c}\text { DOF de N } \\
\text { kg ha }^{-1}\end{array}$ & $\begin{array}{c}\text { Rendimiento } \\
\text { máximo } \\
\text { t ha }^{-1}\end{array}$ & $\begin{array}{c}\text { DOE de N } \\
\text { kg ha- }^{-1}\end{array}$ & $\begin{array}{c}\text { Rendimiento } \\
\text { óptimo } \\
\text { t ha }^{-1}\end{array}$ \\
\hline \multicolumn{7}{|c|}{ Temporada 2002-2003 } \\
\hline FNC & $550^{1}$ & 100.000 & 311 & 10,4 & 253 & 10,21 \\
\hline Urea & $350^{1}$ & 100.000 & 311 & 10,4 & 274 & 10,35 \\
\hline \multicolumn{7}{|c|}{ Temporada 2003-2004 } \\
\hline $\mathrm{FNC}$ & $522^{2}$ & 100.000 & 292 & 9,4 & 239 & 9,18 \\
\hline Urea & $365^{2}$ & 100.000 & 292 & 9,4 & 255 & 9,29 \\
\hline
\end{tabular}

${ }^{1}$ Valores comerciales de abril de 2003.

${ }^{2}$ Valores comerciales de abril de 2004.

Cuadro 5. Efecto de la dosis de $\mathrm{N}$ sobre los parámetros de acidez del Andisol serie Vilcún a la cosecha del trigo cv. Dollinco-INIA. Carillanca, marzo 2003 y 2004.

Table 5. Effect of $\mathrm{N}$ level on acidity parameters of Andisol series Vilcun at harvest of wheat cv. Dollinco-INIA. Carillanca, March 2003 and 2004.

\begin{tabular}{rcccc}
\hline $\begin{array}{r}\mathbf{N} \text { anual } \\
\mathbf{k g ~ h a}^{-1}\end{array}$ & pH agua & $\begin{array}{c}\text { Ca intercambio } \\
\mathbf{c m o l}_{+} \mathbf{k g}^{-\mathbf{1}}\end{array}$ & $\begin{array}{c}\text { Al intercambio } \\
\mathbf{c m o l}_{+} \mathbf{k g}^{-\mathbf{1}}\end{array}$ & $\begin{array}{c}\text { Saturación de Al } \\
\mathbf{\%}\end{array}$ \\
\hline Marzo de 2003 & & & & \\
0 & $6,01 \pm 0,04$ & $9,82 \pm 0,38$ & $0,01 \pm 0,005$ & $0,10 \pm 0,06$ \\
250 & $6,01 \pm 0,05$ & $10,48 \pm 0,32$ & $0,02 \pm 0,009$ & $0,13 \pm 0,09$ \\
300 & $6,10 \pm 0,08$ & $11,16 \pm 0,34$ & $0,01 \pm 0,003$ & $0,11 \pm 0,05$ \\
Marzo de 2004 & & & & \\
0 & $5,89 \pm 0,06$ & $5,60 \pm 0,24$ & $0,05 \pm 0,004$ & $0,71 \pm 0,07$ \\
250 & $5,82 \pm 0,08$ & $5,36 \pm 0,29$ & $0,05 \pm 0,007$ & $0,72 \pm 0,04$ \\
300 & $5,82 \pm 0,03$ & $5,49 \pm 0,27$ & $0,06 \pm 0,005$ & $0,87 \pm 0,08$ \\
\hline
\end{tabular}


Cuadro 6. Efecto de la dosis de $\mathbf{N}\left(\mathrm{kg} \mathrm{ha}^{-1}\right)$ sobre el peso hectolitro $\left(\mathrm{kg} \mathrm{hL}^{-1}\right)$ de grano de trigo $\mathrm{cv}$. Dollinco-INIA sembrado en invierno. Carillanca, 2002-2003 y 2003-2004.

Table 6. Effect of $\mathrm{N}$ level $\left(\mathrm{kg} \mathrm{ha}^{-1}\right)$ on the hectolitre weight $\left(\mathrm{kg} \mathrm{hL}^{-1}\right)$ of wheat grain cv. Dollinco-INIA sowing at winter. Carillanca, 2002-2003 and 20032004.

\begin{tabular}{ccc}
\hline N anual & \multicolumn{2}{c}{ Peso hectolitro } \\
\cline { 2 - 3 } & $\mathbf{2 0 0 2 - 2 0 0 3}$ & $\mathbf{2 0 0 3 - 2 0 0 4}$ \\
\hline 0 & $80,0 \mathrm{~d}$ & $80,7 \mathrm{~b}$ \\
150 & $80,6 \mathrm{~cd}$ & $81,6 \mathrm{ab}$ \\
200 & $81,6 \mathrm{bc}$ & $81,3 \mathrm{ab}$ \\
250 & $82,4 \mathrm{ab}$ & $81,8 \mathrm{ab}$ \\
300 & $82,7 \mathrm{a}$ & $82,2 \mathrm{a}$ \\
\hline
\end{tabular}

Medias con letras diferentes indican diferencias significativas según el Test de Tukey $(\mathrm{P}<0,05)$.

valores de peso hectolitro se consideraron altos y adecuados al cv. Dollinco-INIA (Hewstone y Jobet, 2001).

\section{CONCLUSIONES}

En Andisoles de la Región de La Araucanía, Chile, sometidos a rotaciones intensivas de cultivos anuales, las DOE para Dollinco-INIA se estimaron en 253 (2002-2003) y 239 (2003-2004) $\mathrm{kg} \mathrm{ha}^{-1}$ de N, las cuales resultaron más eficientes desde un punto de vista productivo, económico y sustentable.
Las EUN ( $\mathrm{kg}$ grano producido $\mathrm{kg}^{-1} \mathrm{~N}$ suministrado) calculadas para el cv. Dollinco-INIA fueron altas y fluctuaron entre 57,3 y 34,8 (2002-2003) y entre 52,7 y 31,3 (2003-2004). Las EUN asociadas a las DOE de $\mathrm{N}$ fueron 40,3 y 38,4 en ambas temporadas. Estos valores indican que el manejo del N-fertilizante durante el ciclo de desarrollo del cultivo fue muy eficiente.

El peso hectolitro del cv. Dollinco-INIA se incrementó con el N (2002-2003), aunque sin alcanzar diferencias entre las dosis más altas $(82,4$ y 82,7$)$. En la siguiente temporada, las diferencias de peso hectolitro entre dosis de $\mathrm{N}$ fueron menores y sólo se diferenciaron en las dosis extremas. En ambas temporadas los valores de peso hectolitro fueron altos y adecuados para este cv. de trigo.

No hubo cambios significativos en los valores de acidez residual del suelo ( $\mathrm{pH}$ en agua, $\mathrm{Ca}$ y $\mathrm{Al}$ de intercambio, y saturación de Al), atribuibles a las dosis anuales de $\mathrm{N}$ aplicadas como FNC.

\section{RECONOCIMIENTO}

Los autores agradecen al Ingeniero de Ejecución Agrícola Sr. Carlos Toro C., del Centro Regional de Investigación Carillanca, por su valiosa colaboración en el establecimiento y gestión de los experimentos de campo.

\section{LITERATURA CITADA}

AFIPA. 2006. Manual fitosanitario 2006-2007. 1160 p. Asociación Nacional de Fabricantes e Importadores de Productos Fitosanitarios Agrícolas (AFIPA), Santiago, Chile.

Campbell, C.A., D.R. Cameron, W. Nicholaichuk, and H.R. Davidson. 1977. Effect of fertilizer N and soil moisture on growth, $\mathrm{N}$ content, and moisture use by spring wheat. Can. J. Soil Sci. 57:289-310.

Campillo, R., y J. Rodríguez. 1984. Efecto acidificante de las transformaciones de la urea en dos andisoles de la Región de Los Lagos. Agric. Téc. (Chile) 44:131-138. Campillo, R., y A. Sadzawka. 2006. La acidificación de los suelos. Origen y mecanismos involucrados. In R. Campillo (ed.) Manejo de los recursos naturales en el sistema de incentivos para la recuperación de suelos degradados de la Araucanía. Serie Actas No 38. p. 4460. Instituto de Investigaciones Agropecuarias, Centro Regional de Investigación Carillanca, Temuco, Chile.
CIREN. 2002. Descripciones de suelos. Materiales y símbolos. Estudio Agrológico IX Región. Publicación CIREN No 122.360 p. Centro de Información de Recursos Naturales (CIREN), Santiago, Chile.

Clarke, J.M., C.A. Campbell, H.W. Cutforth, R.M. DePauw, and G.E. Wilkleman. 1990. Nitrogen and phosphorus uptake, translocation, and utilization efficiency of wheat in relation to environment and cultivar yield and protein levels. Can. J. Plant Sci. 70:965-977.

Díaz, B.K., R. Ortega, y J. Riquelme. 2002. Mejores prácticas de manejo para la fertilización en precordillera. In B.K. Díaz (ed.). Mejores prácticas de manejo. Cultivos tradicionales de la zona Centro Sur de Chile. Boletín INIA No 75. p. 71-96. Instituto de Investigaciones Agropecuarias, Centro Regional de Investigación Quilamapu, Chillán, Chile. 
Fowler, D.B., J. Brydon, B.A. Darroch, M.H. Entz, and A.M. Johnston. 1990. Environment and genotype influence on grain protein concentration of wheat and rye. Agron. J. 82:666-674.

Fundación Chile. 2005. Una nueva visión para el sector triguero en Chile. 100 p. Fundación Chile, Santiago, Chile.

Hewstone, C., y C. Jobet. 2001. Dollinco-INIA: trigo alternativo para el sur de Chile. Agric. Téc. (Chile) 61:102-104.

Moll, R.H., E.J. Kamprath, and W.A. Jackson. 1982. Analysis and interpretation of factors which contribute to efficiency of nitrogen utilization. Agron. J. 74:562-564.

Ortiz-Monasterio, J.I., K.D. Sayre, S. Rajaram, and M. McMahon. 1997. Genetic progress in wheat yield and nitrogen use efficiency under four nitrogen rates. Crop Sci. 37:898-904.

ODEPA. 2005. Estadísticas agropecuarias. Cultivos anuales, frutales y hortalizas. Ministerio de Agricultura, Oficina de Estudios y Políticas Agrarias (ODEPA), Santiago, Chile.

Parodi, P. 2003. Mayor eficiencia en el uso del nitrógeno puede aumentar la rentabilidad del trigo y hacerlo más amigable hacia el ambiente. p. 275-283. In Kohli, M.M., M. Díaz, y M. Castro (eds.) Seminario Internacional Estrategias y Metodologías Utilizadas en el Mejoramiento de Trigo, La Estanzuela, Uruguay. 8-11 de octubre de 2001.CIMMYT-INIA, Colonia, Uruguay.

Raun, W.R., and G.V. Johnson. 1999. Improving nitrogen use efficiency for cereal production. Agron. J. 91:357363.
Rebolledo, H. 1999. Estimación de modelos de regresión a experimentos de fertilización y obtención de dosis óptimas económicas de insumos agrícolas. 55 p. Universidad Autónoma Chapingo, Departamento de Suelos, Chapingo, México.

Rodríguez, J. 1991. Manual de fertilización. 362 p. Colección en Agricultura. Pontificia Universidad Católica de Chile, Facultad de Agronomía, Santiago, Chile.

Rouanet, J., I. Pino, A.M. Parada, y A. Nario. 2001. Efecto de tres sistemas de manejo de suelo en la eficiencia fisiológica de uso de nitrógeno fertilizante en trigo. Agric. Téc. (Chile) 61:459-469.

Sadzawka, A., M.A. Carrasco, R. Grez, M.L. Mora, H. Flores, y A. Reaman. 2006. Métodos de análisis recomendados para los suelos de Chile. Revisión 2006. Serie Actas INIA No 34. 164 p. Instituto de Investigaciones Agropecuarias, Centro Regional de Investigación La Platina, Santiago, Chile.

SAS Institute. 1990. SAS/STAT user's guide: statistic. Versión 6. $4^{\text {th }}$ ed. SAS Institute, Cary, North Carolina, USA.

Suárez, D. 1994. Uso de cales y fertilizantes en praderas de la zona sur. p. 39-65. In L. Latrille (ed.) Producción Animal Serie B N ${ }^{\circ}$ 18. Universidad Austral de Chile, Instituto de Producción Animal, Valdivia, Chile.

Volke, H.V. 1982. Optimización de insumos de la producción en la agricultura. $61 \mathrm{p}$. Colegio de Postgraduados, México D.F., México.

Zadoks, J.C., T.T. Chang, and C.F. Zonzak. 1974. A decimal code for the growth stages of cereals. Weed Res. 14:415-421. 